\title{
Mid-IR spectro-imaging observations with the ISOCAM CVF: Final reduction and archive ${ }^{\star, \star \star}$
}

\author{
F. Boulanger ${ }^{1}$, R. Lorente ${ }^{2}$, M. A. Miville Deschênes ${ }^{1,3}$, A. Abergel ${ }^{1}$, J. A. D. L. Blommaert ${ }^{4}$, \\ D. Cesarsky ${ }^{1,5}$, K. Okumura ${ }^{1,6}$, M. Pérault ${ }^{7}$, and W. Reach ${ }^{8}$ \\ ${ }^{1}$ Institut d'Astrophysique Spatiale, Université Paris Sud, Bât. 121, 91405 Orsay Cedex, France \\ e-mail: francois.boulanger@ias.u-psud.fr \\ 2 ISO Data Centre, Astrophysics Division, ESA, Villafranca del Castillo, Spain \\ ${ }^{3}$ Canadian Institute for Theoretical Astrophysics, Toronto, Canada \\ 4 Instituut voor Sterrenkunde, KU Leuven, Belgium \\ 5 Max-Planck-Institut für extraterrestrische Physik, Garching, Germany \\ ${ }^{6}$ Service d'Astrophysique, CEA/DAPNIA, Saclay, France \\ 7 LERMA/ENS, Paris, France \\ ${ }^{8}$ Spitzer Science Center, Caltech, Pasadena, USA \\ Received 22 January 2004 / Accepted 20 January 2005
}

\begin{abstract}
Mid-IR $(5-17 \mu \mathrm{m})$ Spectro-Imaging observations towards several hundred sky positions were obtained with the Infrared Space Observatory Camera (ISOCAM), and a Circular Variable Filter (CVF) that provided spectral resolution $R=$ $\lambda / \Delta \lambda \sim 45$ over a $3^{\prime} \times 3^{\prime}$ field (for 6 , and $12^{\prime \prime}$ pixels, proportionally smaller for the observations carried with the 3 , and $1.5^{\prime \prime}$ pixels). The wavelength range includes dust bands - in particular several of the aromatic carbon bands - fine structure lines from ionized gas, and $\mathrm{H}_{2}$ rotational lines. The observed fields comprise nearby and distant galaxies, Galactic and Extragalactic star forming regions, the Galactic diffuse emission, nearby molecular clouds, infrared cirrus, young stellar objects, evolved stars, and Solar System targets. We present the final data reduction procedure that improves on the standard pipeline reduction in several ways, mainly the subtraction of zodiacal light, that of stray light associated with the uniform, most often dominant, emission component and the correction of a pixel dependent wavelength shift. We also propose a correction of the largest astrometry errors introduced by the optics. We have processed most of the ISOCAM CVF observations and made the results available on the ISO archive for public use. The processed data may be routinely used over the full sensitivity range of the instrument down to a brightness as faint as a few \% of the zodiacal emission. For extended emission, the CVF observations represent a data base with a lasting value which can serve many scientific goals and motivate follow-up observations in particular with the Spitzer Space Telescope spectrometer.
\end{abstract}

Key words. ISM: lines and bands - instrumentation: spectrographs - astronomical data bases: miscellaneous - infrared: general

\section{Introduction}

With the ISO camera (ISOCAM) carrying a Circular Variable Filter (CVF), close to six hundred spectro-imaging observations of a variety of objects were obtained over the wavelength range 5 to $17 \mu \mathrm{m}$ rich in dust features and gas lines. These data were first used to study the spatial distribution of the aromatic carbon dust features, ionized gas, and $\mathrm{H}_{2}$ lines in star forming regions (e.g. Cesarsky et al. 1996a,b; Boulanger et al. 1996). Motivated by the goal of analyzing extended emission in less excited regions, we gathered the necessary understanding of

^ Based on observations with ISO, an ESA project with instruments funded by ESA member states (especially the PI countries: France, Germany, The Netherlands and the UK) and with the participation of ISAS and NASA.

$\star \star$ Appendix A is only available in electronic form at http://www. edpsciences.org the instrument to be able to subtract zodiacal emission, and associated stray light. Preliminary applications of this work have already been reported where we obtained mid-IR spectra of the Helix molecular hydrogen emission (Cox et al. 1998), the zodiacal light (Reach et al. 2003), the Galactic diffuse emission, cirrus clouds (Boulanger et al. 2000), and a low brightness molecular cloud in the Small Magellanic Cloud (Reach et al. 2000).

The full data reduction process that we have developed over the years is now complete, and presented in this paper. The main improvements in the data reduction are subtraction of the zodiacal emission, and associated stray light (Sects. 3, and 4). We also improved the wavelength calibration, and astrometry (Sects. 5, and 6). The results are illustrated on a few examples. Selected observations were analyzed to quantify all sources of photometric uncertainties (Sect. 7). We reprocessed all the ISOCAM-CVF observations with results publicly available in the ISO archive (Appendix A). 


\section{Initial processing steps}

CVF spectroscopic observations were performed by recording sky images over a given range of CVF wheel positions (i.e. wavelengths). Reduction of these data cubes is outlined in the flow diagram of Fig. 1. The first (standard) steps in the data reduction consist of data deglitching, dark subtraction, and correction for the detector gain variations with changes in illumination. These steps are the same for the ISOCAM imaging data mode, and are described in Coulais \& Abergel (2000), Miville et al. (2000), and the ISOCAM Handbook (Blommaert et al. 2003). We made only one minor correction to these first steps in data reduction. To match the zodiacal brightness at $5 \mu \mathrm{m}$ in high Galactic latitude fields, it was found necessary to add a small systematic offset to the raw data. We interpret this correction as a systematic signal residual in dark observations that was not corrected for when producing the ISOCAM dark model (ISOCAM Handbook).

All consecutive images taken at a given CVF wheel position are averaged together to produce a data cube with one image per CVF position (i.e. wavelength). If the data set includes more than one spectral scan, these are not averaged together so that the users can identify, and quantify residual memory effects. The data cube is then photometrically calibrated by applying the photometric spectral response of the ISOCAM CVF derived from observations of calibrations stars (Blommaert et al. 2001a,b).

After these basic data reduction steps, we compared our output with the standard ISOCAM standard pipeline (Off Line Processing v10.0). We found a difference of a few $\%$ that comes from the fact that we use a single flat field for all wavelengths, rather than a flat-field slightly different for different wavelength ranges, in the pipeline.

In the following 4 sections 3 to 6 , we describe additional data reduction steps specific to the CVF: correction for stray light associated with the uniform component of the emission (Sect. 3), zodiacal light subtraction (Sect. 4), wavelengths calibration (Sect. 5), and projection on celestial coordinates (Sect. 6).

\section{Stray light characterization, and correction}

In the CVF spectroscopic mode, reflections between the ISOCAM detector, and the filter wheel cause stray light in the images. This reflected light is seen as ghosts in the case of point sources. For extended emission, the stray-light that is spatially structured, and wavelength dependent affects the photometric calibration in a complex way. We did not reach a sufficient understanding of the reflections to achieve full correction of stray light. Available data allowed us to characterize, and correct the stray light associated with the background illumination, in particular the zodiacal light for observations performed with 6 , and $12^{\prime \prime}$ pixels. The stray light is not that important a problem for observations over a smaller field with the smaller pixels.

To formulate the correction process, we decompose the sky brightness cube in a constant term $B(\lambda)$ and a structure cube

$\operatorname{Sky}(i, j, \lambda)=B(\lambda)+\operatorname{Str}(i, j, \lambda)$.

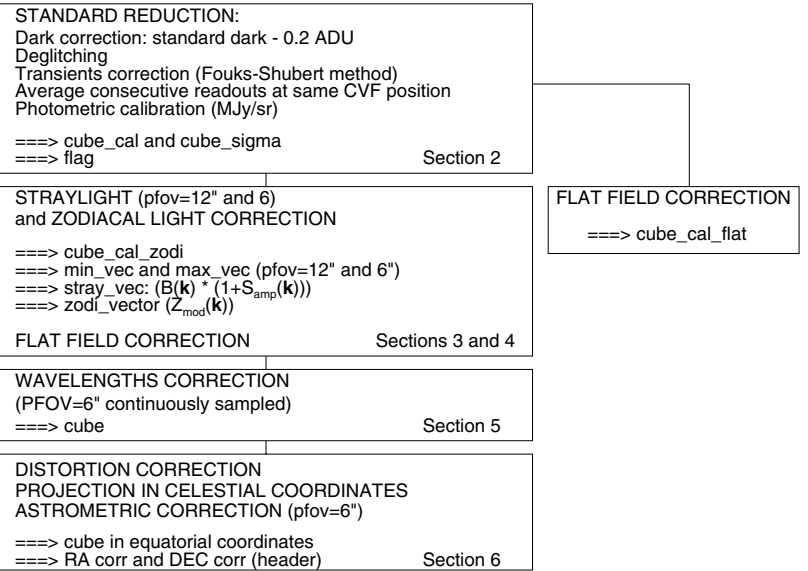

Fig. 1. Data reduction steps, and corresponding paper sections. The cube produced after flat fielding on the side of the main data stream corresponds to output of the standard reduction pipeline. The outputs of the new processing are described in Appendix A.

$B(\lambda)$ is the minimum value of sky brightness over the ISOCAM field, and $\operatorname{Str}(i, j, \lambda)$, the brightness structure above this value which depends on wavelength, and sky position. By definition the minimum of the Str images is 0 at all wavelengths. We consider only the stray light associated with B, and ignore the stray light associated with the structure term of the emission. Within this limitation, the CVF data cube can be written

$$
\begin{aligned}
\operatorname{Data}(i, j, \lambda)= & \operatorname{FF}(i, j) *\left[B(\lambda) *\left(1+S_{\text {cube }}(i, j, \lambda)\right)\right. \\
& +\operatorname{Str}(i, j, \lambda)],
\end{aligned}
$$

where FF is the ISOCAM flat field including the detector response, and the optical vignetting, $\lambda$ the wavelength, $i$, and $j$ the camera pixel indices, and $S_{\text {cube }}$ the stray light contribution. FF is normalized such that for each image, the mean value over the central (unvignetted) part of the array is 1 . We use the standard ISOCAM library flat field determined from calibration observations of cirrus free fields with the wide $12 \mu \mathrm{m}$ filter ( $L W 10)$. In the filter's data, there is no evidence for a significant, and systematic variation in the flat field with wavelength. A single wavelength-independent ISOCAM flat field is thus used. In the following subsections we describe how we estimated $S_{\text {cube }}(i, j, \lambda)$ in Eq. (2), i.e. the amplitude, and the spatial distribution of the stray light for uniform illumination.

\subsection{Stray light amplitude versus wavelength}

CVF observations have been performed with point sources, over a large set of positions over the camera array for a few wavelengths, and for all wavelengths at a few array positions to characterize the position, and amplitude of the ghosts (Okumura et al. 1998; Okumura 2001). Okumura et al. combined these observations to estimate the amplitude of the stray light, averaged over the array, as a function of wavelength $S_{\text {amp }}(\lambda)$. In the following we use $S_{\text {amp }}(\lambda)$ as the mean value of $S_{\text {cube }}(i, j, \lambda)$ averaged over the camera pixels $i$, and $j$.

For an observation on a field with no structure we then have: $\langle\text { Data }\rangle_{\text {array }}(\lambda)=B(\lambda) \times\left(1+S_{\text {amp }}(\lambda)\right)$; in this case, 
the CVF photometry can be corrected from stray radiation by dividing the signal by $1+S_{\text {amp }}(\lambda)$. We validated this correction by comparing $\mathrm{CVF}$, and filter measurements on calibration fields where the sky emission is dominated by a uniform contribution from the zodiacal light. The images taken with the CAM filters are not hampered by reflections because the filters were tilted with respect to the detector. We thus used the filter data as a photometric reference. After division of the CVF data by $\left(1+S_{\text {amp }}(\lambda)\right)$, we found a satisfactory match between $\mathrm{CVF}$, and filter measurements (Blommaert et al. 2001a). The ISOCAM filters, and CVF measurements match the DIRBE zodiacal emission model (Kelsall et al. 1998) within differences of about $10 \%$ which we believe to be significant departures from the zodiacal light spectrum with respect to the model (see Fig. 2, and Reach et al. 2003).

\subsection{Stray light distribution over the array}

In order to determine distribution of the stray light over the array, we made use of CVF data on cirrus free fields at high galactic latitude: 14 observations with $6^{\prime \prime}$ pixels, and 3 for $12^{\prime \prime}$ pixels. At high galactic latitude the zodiacal light is generally the dominant source of extended emission. Quantitatively, where the galactic IRAS $100 \mu \mathrm{m}$ brightness is smaller than a few $\mathrm{MJy} / \mathrm{sr}$, the mid-IR galactic dust emission represents $1-2 \%$ of the total emission at the position of the $6.2 \mu \mathrm{m}$ PAH feature, and less at longer wavelengths where the zodiacal emission is brighter. The zodiacal light may be assumed to be uniform over the $3^{\prime}$ field, so that for our selected fields we can rewrite Eq. (2) as follows:

$\operatorname{Data}(i, j, \lambda)=\operatorname{FF}(i, j) * Z(\lambda) *\left(1+S_{\text {cube }}(i, j, \lambda)\right)$

where $Z(\lambda)$ is the zodiacal light spectrum at the position, and time of the observation.

For each of the observations we computed the ratio data cube Data $(i, j, \lambda) / Z_{\text {mod }}(\lambda)$, where $Z_{\text {mod }}(\lambda)$ is the zodiacal light spectrum computed with the zodiacal model developed for, and fitted on the Diffuse Infrared Background Experiment (DIRBE) observations (Kelsall et al. 1998). This model accounts for the mid-IR amplitude, and color temperature variations with ecliptic coordinates within the range of solar elongations of ISO observations. $Z_{\text {mod }}$ is used to normalize variations in zodiacal emission from one observation to another. The normalized data cubes can be written:

$$
\text { Data } \begin{aligned}
/ Z_{\mathrm{mod}} & =\mathrm{FF}(i, j) * Z(\lambda) / Z_{\mathrm{mod}}(\lambda) *\left(1+S_{\text {cube }}(i, j, \lambda)\right) \\
& =\mathrm{FF}(i, j) * \mathrm{Cal}_{\mathrm{vec}}(\lambda) * \mathrm{Cal}_{\text {cube }}(i, j, \lambda)
\end{aligned}
$$

where $\operatorname{Cal}_{\mathrm{vec}}(\lambda)=Z(\lambda) *\left(1+S_{\mathrm{amp}}(\lambda)\right) / Z_{\mathrm{mod}}(\lambda)$ is a wavelength dependent vector, and $\mathrm{Cal}_{\text {cube }}(i, j, \lambda)=\left(1+S_{\text {cube }}(i, j, \lambda)\right) /(1+$ $S_{\text {amp }}(\lambda)$ ) is a normalized cube characterizing the spatial distribution of the stray light over the array as a function of $\lambda$. $\mathrm{Cal}_{\mathrm{vec}}$ includes the stray light term $\left(S_{\text {amp }}(\lambda)\right)$, and a true difference between the zodiacal light spectrum, and the DIRBE model $\left(Z(\lambda) / Z_{\text {mod }}(\lambda)\right.$ displayed in Fig. 2), which has been interpreted as a signature of emission from small silicate interplanetary particles (Reach et al. 2003). $\mathrm{Cal}_{\mathrm{vec}}$, and $\mathrm{Cal}_{\text {cube }}$ which are computed for each observation. agree within variations smaller than

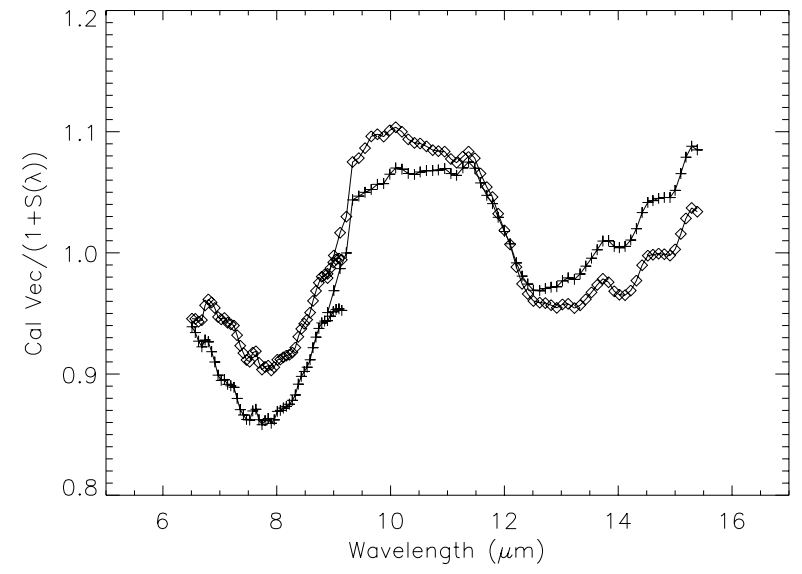

Fig. 2. Calibration vector, $\mathrm{Cal}_{\text {vec }}$ (see text), corrected for the stray light contribution. This vector represents the mean ratio between the CVF, and the DIRBE model spectra of the zodiacal light. Plus signs, and diamonds represent the two scanning directions: down, and up in wavelengths respectively. The small difference between the two curves is caused by a residual memory effect not fully corrected for by the transient correction program.

a few $\%$. To reduce the data noise, we computed the mean values of $\mathrm{Cal}_{\mathrm{vec}}$, and $\mathrm{Cal}_{\text {cube }}$ over the selected set of observations.

$\mathrm{Cal}_{\text {cube }}$, and $\mathrm{Cal}_{\mathrm{vec}}$ are obtained independently for the 6 , and $12^{\prime \prime}$ pixels. The $\mathrm{Cal}_{\mathrm{vec}}$ vectors are quite similar for the two pixel sizes but the $\mathrm{Cal}_{\text {cube }}$ cubes do differ.

\subsection{Stray light correction}

With $\mathrm{Cal}_{\text {cube }}$, and $\mathrm{Cal}_{\mathrm{vec}}$, Eq. (2) may be written:

$\operatorname{Data}(i, j, \lambda)=\mathrm{FF}(i, j) *\left[B(\lambda) * \mathrm{Cal}_{\text {cube }} * \mathrm{Cal}_{\mathrm{vec}}\right.$

$$
\left.* Z_{\bmod } / Z(\lambda)+\operatorname{Str}(i, j, \lambda)\right] .
$$

Since the minimum value of the Str cube is 0 for each $\lambda$, we compute $B(\lambda)$ using the following expression:

$$
\begin{array}{r}
B(\lambda)=\operatorname{Min}\left[\operatorname{Data}(i, j, \lambda) /\left(\mathrm{FF}(i, j) * \operatorname{Cal}_{\text {cube }}(i, j, \lambda)\right)\right] \\
/\left(1+S_{\mathrm{amp}}(\lambda)\right) .
\end{array}
$$

To reduce the effect of noise, the data are spatially smoothed before computing this minimum value. The second term of the Sky cube expression in Eq. (1), the Str cube, then follows from the expression:

$$
\begin{aligned}
\operatorname{Str}(i, j, \lambda)= & \operatorname{Data}(i, j, \lambda) / \operatorname{FF}(i, j) \\
& -B(\lambda) *\left(1+S_{\text {amp }}(\lambda)\right) * \mathrm{Cal}_{\text {cube }} .
\end{aligned}
$$

This correction is only applied to observations performed with 6 , and $12^{\prime \prime}$ pixels.

\section{Zodiacal light subtraction}

To subtract the zodiacal light contribution $Z(\lambda)$ to the measured emission, $Z_{\text {mod }}(\lambda) * \mathrm{Cal} \mathrm{Vec} /\left(1+S_{\mathrm{amp}}(\lambda)\right)$ is subtracted from $B(\lambda)$ obtained from Eq. (6). We then obtain a data cube 

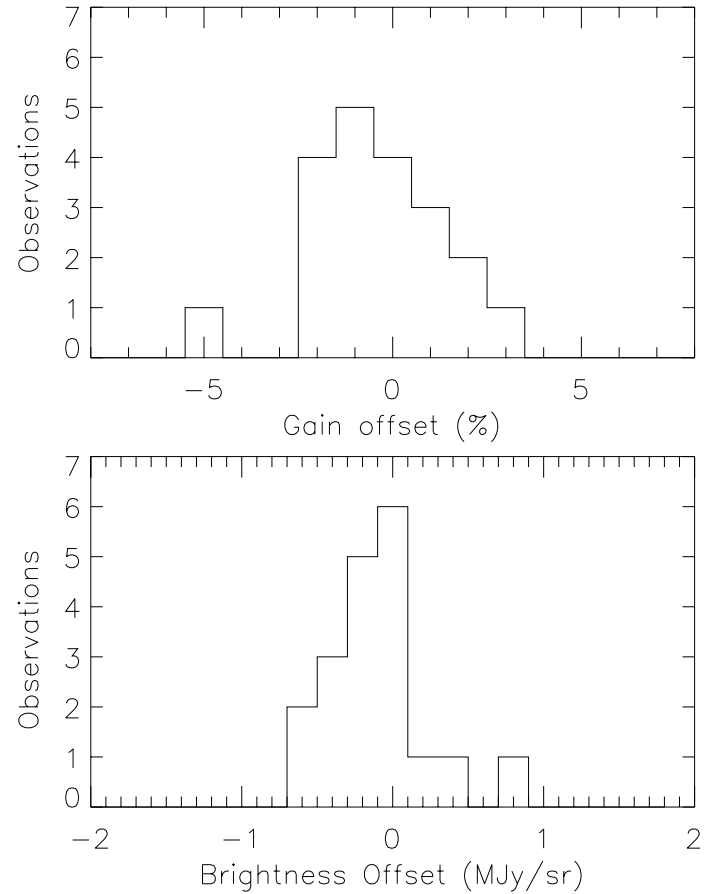

Fig. 3. Histogram of gain offsets from 1, and brightness offsets for a set of 21 observations at high galactic latitude. The gains, and offsets were determined by linearly fitting the measured spectra to the expected spectrum based on the DIRBE zodiacal model. The standard deviations are $1.7 \%$, and $0.4 \mathrm{MJy} / \mathrm{sr}$ for the gains, and offsets, respectively.

corrected for zodiacal light and for stray light associated with the background emission:

$$
\begin{aligned}
& \operatorname{Sky}(i, j, \lambda)-Z(\lambda)=\operatorname{Data}(i, j, \lambda) / \mathrm{FF}(i, j)-B(\lambda) \\
& *\left(1+S_{\mathrm{amp}}(\lambda)\right) * \mathrm{Cal}_{\mathrm{cube}}+B(\lambda)-\mathrm{Cal}_{\mathrm{vec}} * Z_{\mathrm{mod}} /\left(1+S_{\mathrm{amp}}(\lambda) .(8)\right.
\end{aligned}
$$

The accuracy with which the zodiacal light is subtracted is limited by variations in the gain, and offset of the ISOCAM detector. We quantified these variations with a set of 21 observations of cirrus-free regions at high galactic latitude by comparing the calibrated spectrum with the spectrum derived from the DIRBE model: $Z_{\bmod }(\lambda) * \mathrm{Cal} \mathrm{Vec} /(1+S(\lambda))$. The two spectra are always very close to each other, and gain, and offset variations can be computed through a linear regression. Histograms of the fitted gains, and offsets are presented in Fig. 3. The standard deviation is $1.7 \%$ for the gain, and $0.4 \mathrm{MJy} / \mathrm{sr}$ for the offset.

\section{Wavelength calibration}

In the CVF cubes produced with the standard reduction, the central wavelengths of gas emission lines are slightly shifted from the nominal values with an offset varying smoothly across the array. The wavelength offsets are positive, and range up to $0.1 \mu \mathrm{m}$ for the long wavelength segment $(\lambda>9 \mu \mathrm{m})$, and up to $0.045 \mu \mathrm{m}$ for the short wavelengths $(\lambda<9 \mu \mathrm{m})$. This is a noticeable effect of up to $1 \mathrm{CVF}$ wheel step (about half the spectral resolution). A CVF spectrum $\operatorname{Sky}(i, j, k)$, where $i$ and $j$ refer to the ISOCAM array pixel and $k$ to the CVF filter wheel position can be expressed as

$\operatorname{Sky}(i, j, k)=I\left(i, j, \lambda_{\mathrm{CVF}}(k)+\operatorname{Woff}(i, j, k)\right)$,
Table 1. Wavelengths calibration.

\begin{tabular}{lll}
\hline \hline Target & Lines & Observation TDT \\
\hline Helix & $\mathrm{H}_{2}$, NeIII & 53600403 \\
& $\mathrm{H}_{2}$, NeIII & 53600707 \\
& ArIII, NeIII & 53600604 \\
& NeIII & 75401705 \\
IC 443 & H2 & 83401648 \\
M17 & NeIII & 10402701 \\
30 Dor & ArIII, SIV, NeIII & 68802002 \\
\hline
\end{tabular}

Note: List of observations, and gas lines used for wavelengths calibration of $6^{\prime \prime}$ observations.

where $\lambda_{\mathrm{CVF}}(k)$ is the nominal wavelength for the $k$ position of the CVF wheel and Woff is a pixel, and wavelength dependent offset. Woff needs to be determined to re-intrepolate the spectra over the nominal wavelength vector $\lambda_{\mathrm{CVF}}$.

The list of targets, observations, and gas lines used to empirically determine Woff are listed in Table 1 . We chose targets with no, or only weak, dust features (Helix, IC 443, 30 Dor), and also used the NeIII emission line in M 17 that is well separated from the main dust features. We did not use the $\mathrm{NeII}(12.81 \mu \mathrm{m})$, and $\mathrm{H}_{2} \mathrm{~S}(2)(12.28 \mu \mathrm{m})$ lines that are close to each other. The reference wavelengths for which the Woff values could be determined, are for the short wavelength CVF segment (5 to $9.3 \mu \mathrm{m}$ ): the $\mathrm{S}(4)$ to $\mathrm{S}(7)$ lines of $\mathrm{H}_{2}$ at 5.51, 6.11, 6.91 , and $8.02 \mu \mathrm{m}$, the ArIII line at $8.99 \mu \mathrm{m}$ and for the long wavelengths CVF segment (9.1 to $17 \mu \mathrm{m})$ the $\mathrm{S}(3)$ line of $\mathrm{H}_{2}$ at $9.665 \mu \mathrm{m}$, the SIV line at $10.51 \mu \mathrm{m}$, and the Ne III line at $15.555 \mu \mathrm{m}$. The central wavelengths of the lines were measured with Gaussian fits. To increase the line signal to noise ratio, and eliminate un-flagged glitches, the data were spatially smoothed with a median filter over a $5 \times 5$ pixels box before fitting. For most of the reference wavelengths, at least two independent observations were considered. Independent measurements were averaged with relative weights proportional to the signal to noise ratio squared to provide a set of wavelength correction images for the reference wavelengths 5.51, 6.11, 6.91, 8.02, $8.99,9.665,10.51$, and $15.555 \mu \mathrm{m}$. By comparing independent measurements, we estimate the accuracy of these images to $\sim 0.01 \mu \mathrm{m}$ at long wavelength $(9.5$ to $16 \mu \mathrm{m})$, and $\sim 0.005 \mu \mathrm{m}$ at short wavelengths ( 5 to $9 \mu \mathrm{m}$ ).

We obtained the full cube of offsets Woff $(i, j, k)$ by linearly interpolating between the reference wavelengths. Beyond the extreme ones, corrections were assumed not to vary with $\lambda$. In the data processing, the Woff cube is used to interpolate CVF spectra to the nominal CVF wavelengths $\lambda_{\mathrm{CVF}}$, and produce a cube composed of sky images at fixed wavelengths. This interpolation made with cubic splines does not affect the spectral resolution, and is only applied to fully sampled spectra.

\section{Astrometry}

ISOCAM observations are known to suffer from an astrometry error a few pixels larger than the satellite absolute pointing accuracy, and jitter. These errors are thought to come from 
Table 2. Astrometry of 6 " pixels observations.

\begin{tabular}{lrrcc}
\hline \hline TDT & $\begin{array}{r}\text { Horizontal } \\
\text { shift }\end{array}$ & $\begin{array}{r}\text { Vertical } \\
\text { shift }\end{array}$ & Sources & Vignetting \\
\hline 83800324 & $-2.0 \pm 0.1$ & $0.8 \pm 0.1$ & 7 & $\mathrm{~L}$ \\
83800427 & $-2.1 \pm 0.1$ & $1.2 \pm 0.1$ & 6 & $\mathrm{~L}$ \\
84700430 & $-1.8 \pm 0.3$ & $0.1 \pm 0.2$ & 3 & $\mathrm{~L}$ \\
14801421 & $-1.5 \pm 0.4$ & $1.1 \pm 0.1$ & 3 & $\mathrm{~L}$ \\
15500717 & $-2.3 \pm 0.2$ & $0.4 \pm 0.3$ & 3 & $\mathrm{~L}$ \\
33001007 & $-0.5 \pm 0.0$ & $0.5 \pm 0.1$ & 2 & $\mathrm{C}$ \\
45201111 & $1.0 \pm 0.4$ & $0.4 \pm 0.1$ & 2 & $\mathrm{C}$ \\
15500619 & $-2.0 \pm 0.3$ & $0.3 \pm 0.1$ & 2 & $\mathrm{~L}$ \\
16700323 & $-1.4 \pm 0.1$ & $0.5 \pm 0.0$ & 3 & $\mathrm{~L}$ \\
33001305 & $-0.1 \pm 0.0$ & $0.7 \pm 0.0$ & 2 & $\mathrm{C}$ \\
45601809 & -2.1 & 1.0 & 1 & $\mathrm{~L}$ \\
30801515 & 1.1 & 0.7 & 1 & $\mathrm{C}$ \\
86900783 & $0.0 \pm 0.1$ & $0.6 \pm 0.2$ & 4 & $\mathrm{C}$ \\
87200345 & $1.3 \pm 0.1$ & $0.5 \pm 0.4$ & 2 & $\mathrm{C}$ \\
87200477 & $0.2 \pm 0.3$ & $0.7 \pm 0.2$ & 3 & $\mathrm{C}$ \\
87200346 & $-0.2 \pm 0.2$ & $0.4 \pm 0.0$ & 2 & $\mathrm{C}$ \\
\hline
\end{tabular}

ISOCAM CVF observations with 6" pixels used to evaluate astrometric uncertainties. The 4 th column indicates the number of ISO sources cross-correlated with 2MASS comments. The comments come from the automatic detection of the under illuminated column: $\mathrm{C}$ means that the image is centered (the two edge columns have comparable brightness), and $\mathrm{L}$ that the image is left shifted (the right edge column is under illuminated).

jitter in the positioning of the lens wheel after any optical move (ISO Handbook: Blommaert et al. 2003).

Several ISOCAM CVF fields populated by point sources that could be cross correlated with 2MASS detections were selected in order to characterize the CVF astrometry errors. Results are listed in Tables 2 and 3. For the 6" pixel observations, we find that the horizontal errors are correlated with the vignetting pattern in the images. Large horizontal shifts of $-2 \pm 0.5$ pixels correspond to observations where the right column of the array are under-illuminated (marked L in Table 2). For other observations the horizontal shift is found to be close to 0 or positive. For the vertical shift we find a mean value of 0.6 pixels, and a standard deviation of 0.3 ; it is uncorrelated with the vignetting pattern. The 3 " prov images have been classified by eye. As for the 6" observations, the largest errors $3.2 \pm 0.3$ pixels are found in left shifted images. For the others the horizontal shift is $-0.9 \pm 0.4$ pixels. We studied neither the astrometry of the $1.5^{\prime \prime}$ pixel observations where the satellite pointing becomes a significant source of error, nor those of $12^{\prime \prime}$ observations used only towards fields with diffuse extended emission, for which accurate astrometry is not a concern.

For both the 3, and 6" pixels, we find that the main astrometric error corresponds to horizontal shift over the array correlated with the vignetting pattern at the detector edges. The error can be reduced to less than a pixel by taking advantage of this correlation. For the $6^{\prime \prime}$ pixel observations, where the vignetting pattern was found to be shifted to the left, an astrometric correction corresponding to a 2 pixel horizontal shift was computed. The corrected central coordinates appear in the
Table 3. Astrometry of $3^{\prime \prime}$ pixel observations.

\begin{tabular}{lrrcc}
\hline \hline TDT & $\begin{array}{r}\text { Horizontal } \\
\text { shift }\end{array}$ & $\begin{array}{r}\text { Vertical } \\
\text { shift }\end{array}$ & Sources & Vignetting \\
\hline 14900601 & $-0.9 \pm 0.0$ & $1.4 \pm 0.2$ & 2 & $\mathrm{C}$ \\
33000703 & $-3.0 \pm 0.3$ & $0.5 \pm 0.2$ & 3 & $\mathrm{~L}$ \\
87300701 & -1.1 & 1.0 & 1 & $\mathrm{C}$ \\
70602767 & $-3.6 \pm 0.2$ & $0.1 \pm 0.2$ & 2 & $\mathrm{~L}$ \\
76300157 & -2.9 & 1.8 & 1 & $\mathrm{~L}$ \\
85001301 & -3.0 & 1.3 & 1 & $\mathrm{C}$ \\
32701052 & -0.4 & 0.4 & 1 & $\mathrm{C}$ \\
36401031 & -3.4 & 0.8 & 1 & $\mathrm{~L}$ \\
55501102 & -1.2 & 1.0 & 1 & $\mathrm{C}$ \\
\hline
\end{tabular}

ISOCAM CVF observations with 3" pixels used to evaluate astrometric uncertainties. The comments are as in Table 2 but in this case come from visual assessment.

main header of the data product as keywords "RA corr", and "Dec corr". Before applying these corrections the user must check that the vignetting at the left/right edges of the array is not confused by structured emission because the automatic assessment might in this case give an erroneous answer. For the $3^{\prime \prime}$ observations, no automatic measurement of the vignetting pattern was made. Coordinate correction can be computed from the camera angle (roll angle) provided in the data header.

\section{Assessment of processed data}

\subsection{Comparison with pipeline products}

The impact of the new CVF data reduction depends on the nature of the emission, and the scientific scope of the study. Improvements in the data reduction are the most significant for fields with extended emission fainter than the zodiacal foreground, observed with the 6 or $12^{\prime \prime}$ pixel field of view. The spectra, and spatial structure of such emission can now be studied, thanks to zodiacal light subtraction, and stray light correction. For brighter emission, and/or compact sources the main improvements are the correction of the wavelength shift, and the removal of the stray light associated with the uniform component of the detector illumination.

The new, and pipeline data products are compared in three figures presenting a spectrum of the diffuse galactic emission (Fig. 4), the spectrum of the M 31 stellar bulge (Fig. 5), and images of the $11.3 \mu \mathrm{m}$ emission from dust clouds in the M 31 bulge in Fig. 6 (Cesarsky et al. 1998). In total photometry mode, where the data cube is averaged to produce a mean spectrum of the emission within the camera field of view, dust features with amplitudes of a few \% of the zodiacal light can be measured. In the top spectrum of Fig. 4, the output of the pipeline processing, the features are buried in the zodiacal light emission. With Fig. 5 we show that the new processing also improves differential spectro-photometry of extended emission within the camera field of view. In Fig. 6 we compare images of dust clouds in the $11.3 \mu \mathrm{m}$ dust features seen within the M 31 bulge. The stellar axisymmetric emission has been subtracted. The contours represent the $11.3 \mu \mathrm{m}$ emission obtained 


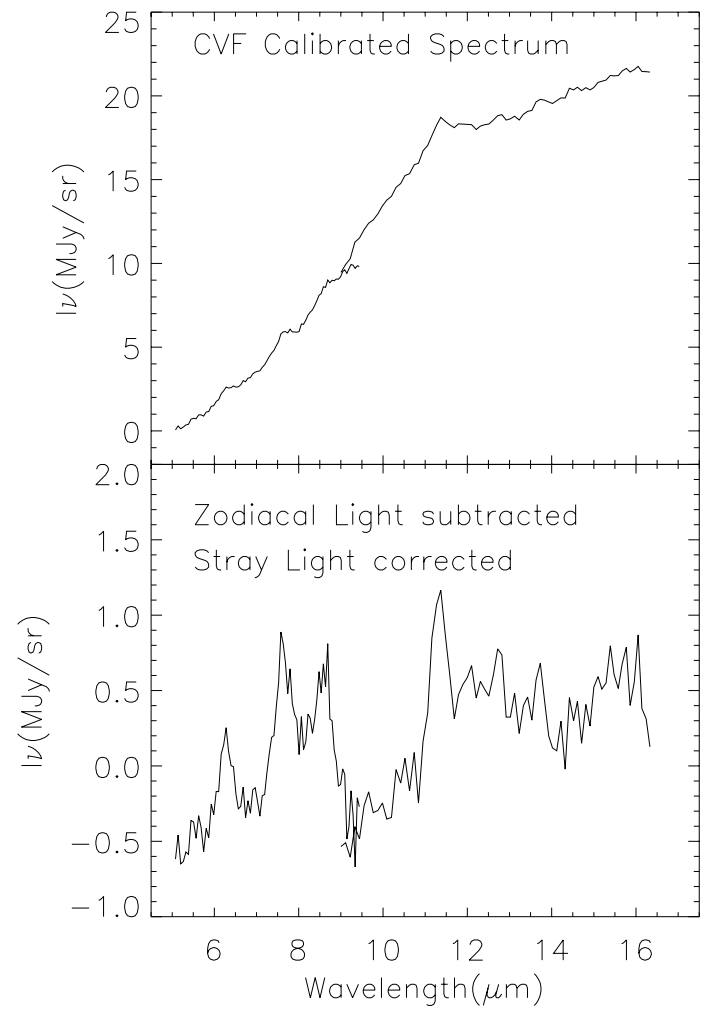

Fig. 4. Spectroscopy of faint extended emission at a local minimum of galactic emission at $l=299.67^{\circ}, b=-16.32^{\circ}$ obtained in $1 \mathrm{~h}$ integration time. The spectrum in the top panel is the result of standard pipeline processing. The bottom spectrum is the one obtained after zodiacal light subtraction, and stray light correction.

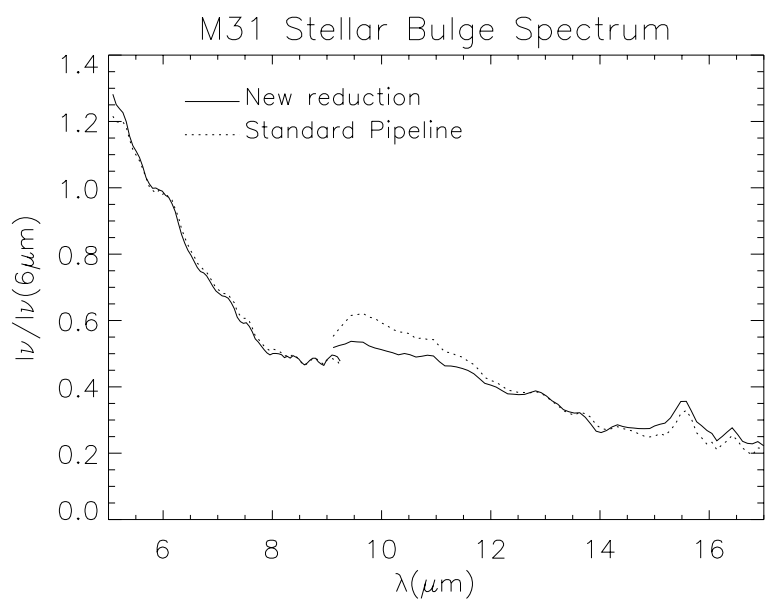

Fig. 5. CVF spectrum of the M 31 stellar bulge with the new reduction (thick solid line), and standard pipeline (dotted line). For each CVF wavelength we linearly fitted the bulge image to that at $6 \mu \mathrm{m}$. The plotted spectra represent the slope of this linear regression.

by subtracting the continuum emission measured from images on each side of the dust feature; the subtraction removes the stray light associated with continuum stellar emission, which is much more intense than that of the dust clouds. The contour thus serves as a reference to identify stray light in the two images. The diffuse emission in the pipeline image seen outside of the contours is stray light corrected in the new reduction.

\subsection{Photometric uncertainties}

\subsubsection{Statistical noise}

The observations at high galactic latitude used to determine the zodiacal light spectrum, and the stray light have also been used to characterize the data noise. We distinguish the spatial noise $\sigma_{\text {spatial }}(\lambda)$ (the standard deviation of the brightness at wavelength $\lambda$ ), and the spectral noise $\sigma_{\text {spectral }}(\lambda)$ (the standard deviation after removal of the linearly interpolated brightness computed from two frames four wavelength steps away on each side). The first noise is relevant for maps of continuum emission while the second one is relevant for imaging emission in gas lines or dust bands after continuum subtraction. The two types of noise are plotted in Fig. 7 for a set of high galactic latitude observations with $60 \mathrm{~s}$ integration time per CVF step, and 6" pixel. A spectral sensitivity about one order of magnitude better than what is shown in Fig. 7 is obtained on the mean emission by averaging all the camera pixels together (e.g. in the diffuse galactic spectrum in Fig. 4).

The difference between spectral, and spatial noises is well accounted for by a flat field noise equal to $2.5 \%$ of the mean brightness. One can obtain the spatial noise from the spectral noise using the following formula:

$$
\begin{aligned}
\sigma_{\text {spatial }}(\lambda)= & \sigma_{\text {spectral }}(\lambda) \\
& *\left[1+\left(2.5 \times 10^{-2} * B(\lambda) / \sigma_{\text {spectral }}(\lambda)\right)^{2}\right]^{0.5}
\end{aligned}
$$

A flat field noise of this amplitude also accounts for the observed increase in spatial noise at a given wavelength with the amplitude of the emission. We believe that the flat field noise is dominated by residuals of detector transients. The flat field noise does not affect the spectral noise, which shows neither systematic dependence on wavelength nor on the amplitude of the emission.

\subsubsection{Systematic uncertainties}

For continuum measurements, one must add 3 contributions to the statistical noise: systematic uncertainties associated with the dark current, zodiacal light subtraction, and residual transient effects. Uncertainties from the dark current, and zodiacal light subtraction are quantified in Fig. 3. The standard deviation found among the observations which were analyzed to characterize the stray light pattern, and the zodiacal emission is $1.7 \%$ for the gain, and $0.4 \mathrm{MJy} / \mathrm{sr}$ for the brightness offset ( $1 \sigma$ values). This last number is interpreted as an uncertainty on the dark subtraction, and needs to be multiplied by (pixelsize $\left./ 6^{\prime \prime}\right)^{2}$ for observations carried out with a pixel size different than $6^{\prime \prime}$.

The photometric uncertainties due to residual transient effects can be assessed by comparing upward, and downward scans when both are available. For the high galactic latitude observations analyzed in Sect. 2, the two curves in Fig. 2 cross each other at about $12 \mu \mathrm{m}$, and the differences for longer, and shorter wavelengths approaches 5\%. Analysis of other observations performed in both scanning directions show that this is a general pattern but that the crossover point, and amplitude of the difference depends on the scanning speed, and sky 

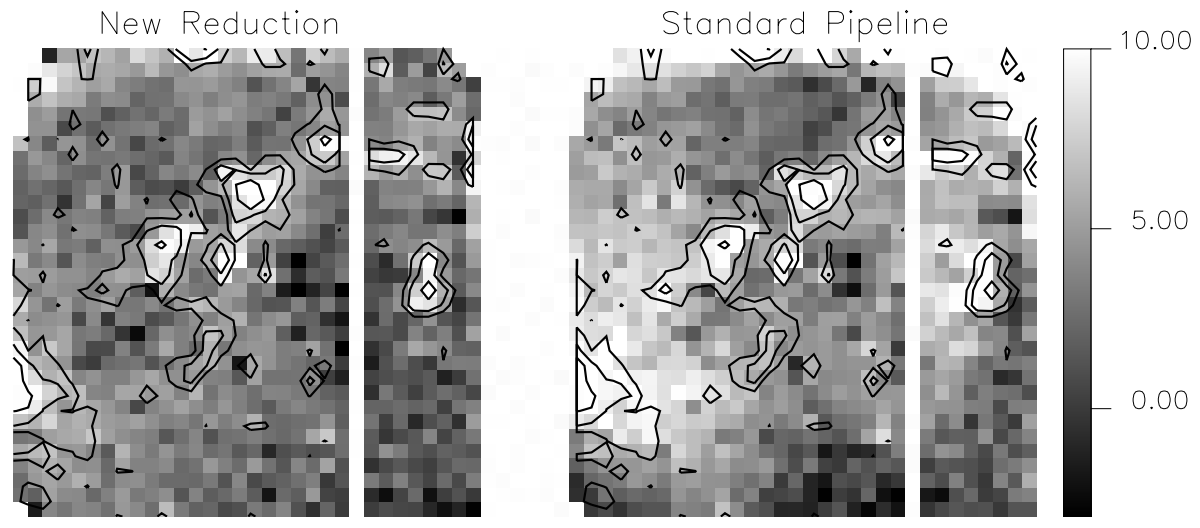

Fig. 6. CVF images at $11.3 \mu \mathrm{m}$ of dust clouds in the M 31 bulge The stellar axisymmetric emission has been subtracted. The contours that serve as a reference represent the $11.3 \mu \mathrm{m}$ emission obtained by subtracting the continuum emission measured from images on each side of the dust feature. The diffuse emission in the pipeline image seen outside of the contours is mostly stray light corrected in the new reduction.

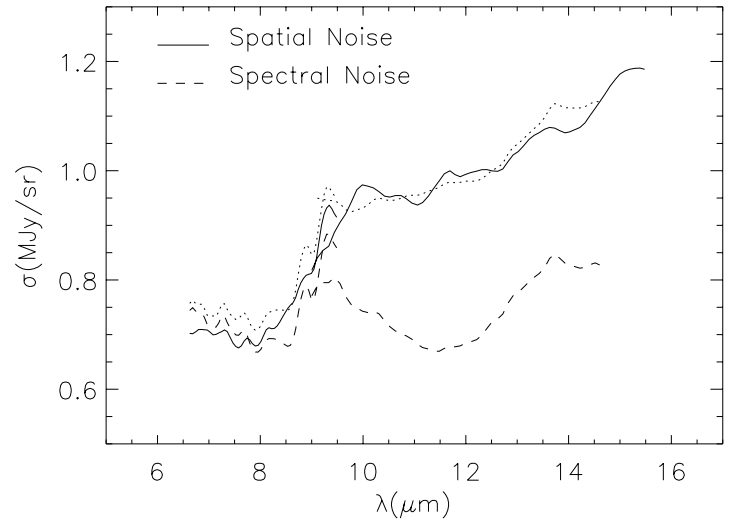

Fig. 7. Noise amplitude computed from a set of high galactic latitude observations with a common integration time per wavelength (i.e. CVF step) of $60 \mathrm{~s}$, and pixel size of $6^{\prime \prime}$. Spectral noise $\sigma_{\text {spectral }}(\lambda)$ is the standard deviation of the difference image data $(i, j, k)-($ data $(i, j, k-$ $4)+\operatorname{data}(i, j, k+4)) / 2$ ), where $i$, and $j$ are the ISOCAM array indices, and $k$ the CVF wheel position index corresponding to the wavelength $\lambda$. The thin dotted line represents spatial noise estimated from the spectral noise using Eq. (10) in the text.

brightness. The amplitude of the effect expressed in \% of sky brightness decreases for increasing brightnesses. The $5 \%$ fraction obtained for spectra at high galactic latitude is thus a maximum difference between upward, and downward scans.

For an observation of extended emission fainter than the zodiacal foreground, carried out at a similar scanning speed to these reference observations $(1.2 \mathrm{~h} / \mathrm{scan})$, the zodiacal light subtraction which takes into account the difference shown in Fig. 2 removes most of the residual transient effect. For observations carried out with a different speed we checked that the difference between upward, and downward scans can be up to a few $\%$ for low sky brightness. It is smaller when the source brightness is larger than the zodiacal light.

\subsection{Stray light}

In the data processing only stray light associated with the uniform component (minimum brightness on the detector) is corrected for. For observations with high signal to noise spatial

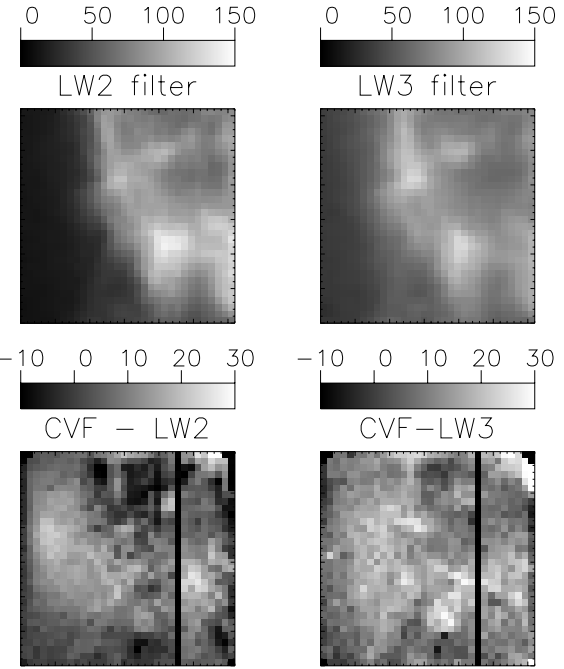

Fig. 8. Difference between filters $(L W 2 \quad(5-8.5 \mu \mathrm{m}), \quad L W 3$ $(12-18 \mu \mathrm{m})$ ), and CVF observations for a $3^{\prime} \times 3^{\prime}$ field north of NGC 2023 within the Orion B images presented by Abergel et al. (2002).

structure, stray light is the main source of error that affects images and absolute photometry. Spectroscopic evidence of uncorrected stray light is the jump in brightness at $9 \mu \mathrm{m}$ between the two CVF segments (see Fig. 5). For point sources, the calibration observations presented by Okumura et al. (1998) can help identify, and discard ghosts. For extended structure, we can only make qualitative statements. For two examples we show the observed difference between filter, and CVF observations (Figs. 8 and 9) after convolving the CVF data with the spectral pass bands of the filters.

In these examples we find that CVF brightness is larger than what is measured with the filter. The amplitude of this effect ranges from 5 to $15 \%$. We believe that this positive difference corresponds to stray light emission. The stray light thus limits the photometry accuracy of extended structures within the camera field of view. Stray light also limits the useful dynamic range of the images. For example, a significant fraction of the signal measured by the CVF in the dark half of the NGC 2023 field is stray light coming from the brighter half of the image. 


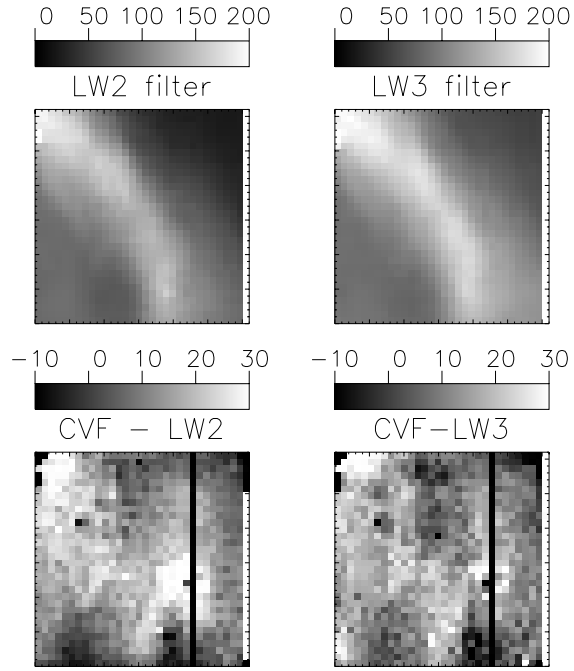

Fig. 9. Difference between filters ( $L W 2$, and $L W 3$ ), and CVF observations for a $3^{\prime} \times 3^{\prime}$ piece of the western interface of the $\rho$ Ophiuchus cloud within the two images presented by Abergel et al. (1996).

In the two fields shown in Figs. 8 and 9), the filter to CVF differences in the low brightness parts of the images amount to a few $\%$ of the peak brightness. Based on these two examples, and a few more fields where CVF, and filter images could be compared, we estimate the maximum useful dynamic range of the CVF images to be about 10 .

\section{Comparison with the Spitzer spectrograph}

For extended emission, the processed CVF observations represent a data base with lasting value serving many scientific goals, and motivating follow-up observations in particular with the Infrared Spectrograph (IRS) on the Spitzer Space Telescope (Houck et al. 2004). The ISOCAM CVF is comparable in wavelength range, and spectral resolution to the short low module of IRS. IRS is more sensitive than the CVF for point sources but for extended emission this gain in sensitivity is reduced by the difference in pixel size (1.8 versus $\left.6^{\prime \prime}\right)$. The sensitivity achieved on extended emission with the ISOCAM CVF is only a factor of about 2 lower than that announced for the same integration time with the short low module of the Spitzer Space Telescope spectrometer IRS. Due to the large number of required pointings, we computed that it will take a factor three more observing time for IRS than with the CVF to cover the same $3^{\prime} \times 3^{\prime}$ field of view with a comparable sensitivity. For extended sources observed with the CVF, the IRS can be used to obtain higher angular resolution on selected parts of the field, and to extend the spectra to longer wavelengths with the Long Low module. CVF observations can also be used in relation to IRAC imaging observations to provide insight into the origin of the emission in the 5.8 , and $8 \mu \mathrm{m}$ IRAC bands.

\section{Summary}

We have presented final processing of the spectro-imaging mid-IR observations obtained with the ISOCAM CVF. Improvements in data reduction are the most significant for fields with extended emission fainter than the zodiacal foreground, observed with 6 or $12^{\prime \prime}$ pixels. Thanks to zodiacal light subtraction, and stray light correction, the spectrum, and spatial structure of these objects can now be easily obtained from the data. The large majority of observations fall in this category. The new reduction makes it possible to extend scientific analysis that have been focused mainly on bright objects to fainter sources, thus probing less excited environments.

We were able to correct the $6^{\prime \prime}$ pixel observations for a spatially dependent shift in the wavelength and to provide a correction of the astrometry shifts introduced by the camera optics based on the vignetting pattern in the images. All of the CVF observations have been re-processed, and are publicly available on the ISO Archive web site. This paper serves as the explanatory supplement to the released data. The reduced data is assessed with a quantitative discussion of systematic uncertainties. We encourage interested scientists to take advantage of this new ISO data product.

Acknowledgements. ISO is an ESA project with instruments funded by ESA Member States, and with the participation of ISAS, and NASA.

\section{References}

Abergel, A., Bernard, J. P., Boulanger, F., et al. 1996, A\&A, 315, L329 Abergel, A., Bernard, J. P., Boulanger, F., et al. 2002, A\&A, 389, 239 Blommaert, J. A. D. L., Boulanger, F., \& Okumura, K. 2001a, ISOCAM CVF Photometry Report

(http://www.iso.vilspa.esa.es/users/expl_lib/ CAM_list.html)

Blommaert, J. A. D. L., Okumura, K., \& Boulanger, F. 2001b, in The Calibration Legacy of the ISO Mission, ed. L. Metcalfe, A. Salama, S. B. Peschke, \& M. F. Kessler, ESA SP-481, 2003, 77

Blommaert, J. A. D. L., Siebenmorgen, R., Coulais, A., et al. 2003, The ISO Handbook, Volume II - CAM - The ISO Camera, version 2.0. Series, ed. T. G. Mueller, J. A. D. L. Blommaert, \& P. Garcia-Lario, ESA SP-1262, European Space Agency (http://www.iso.vilspa.esa.es)

Boulanger, F., Reach, W. T., Abergel, A., et al. 1996, A\&A, 315, L325 Boulanger, F., Abergel, A., Cesarsky, D., et al. 2000, in ISO Beyond Point Sources: Studies of Extended Infrared Emission, ed. R. J. Laureijs, K. Leech, \& M. F. Kessler, ESA-SP 455, 91

Cesarsky, C. J., Abergel, A., Agnèse, P., et al. 1996a, A\&A, 315, L32 Cesarsky, D., Lequeux, J., Abergel, A., et al. 1996b, A\&A, 315, L305 Cesarsky, D., Lequeux, J., Pagani, L., et al. 1998, A\&A, 337, L35

Coulais, A., \& Abergel, A. 2000, A\&AS, 141, 533

Cox, P., Boulanger, F., Huggins, P. J., et al. 1998, ApJ, 495, L23

Houck, J. R., Roellig, T. L., van Cleve, J., et al. 2004, ApJS, 154, 18

Kelsall, T., Weiland, J. L., Franz, B. A., et al. 1998, ApJ, 508, 44

Miville, M. A., Boulanger, F., Abergel, A., \& Bernard, J. P. 2000, A\&AS, 146, 519

Okumura, K. 2001, in The Calibration Legacy of the ISO Mission, ed. L. Metcalfe, A. Salama, S. B. Peschke, \& M. F. Kessler, ESA SP-481, 2003, 211

Okumura, K., Pérault, M., \& Longval, Y. 1998, technical report, Ghosts in ISOCAM images

(http://www.iso.vilspa.esa.es/users/expl_lib/ CAM_list.html)

Reach, W. T., Boulanger, F., Contursi, A., \& Lequeux, J. 2000, A\&A, 361,895

Reach, W. T., Morris, P., Boulanger, F., \& Okumura, K. 2003, Icarus, 164,384 
F. Boulanger et al.: New reduction and archive of ISOCAM CVF observations, Online Material $p 1$

\section{Online Material}


F. Boulanger et al.: New reduction and archive of ISOCAM CVF observations, Online Material p 2

\section{Appendix A: Data in the ISO archive}

\section{A.1. Observed fields}

Almost all of the observations taken in the CVF mode were processed and are in the new archive. We did not process the observations obtained with the SW (Short Wavelengths) detector at $\lambda<5 \mu \mathrm{m}$. Short CVF observations taken on an off position, before and after moving to a bright source, were used for the transient correction but were not kept as entries in the archive. Figure A.1 shows the histograms of the median value and the dynamic range of the extended emission in the field, expressed in units of the zodiacal light at $12 \mu \mathrm{m}$. These histograms show that the large majority of the observations have a median brightness close to, and a dynamic range smaller than or comparable to, the zodiacal emission. These are the observations that benefit most from the new reduction. According to the category given by the observers in their proposals, the CVF data base consists of: $46 \%$ extragalactic fields; $27 \%$ interstellar matter; $21 \%$ stellar physics; $3 \%$ solar system objects; $1 \%$ cosmology; and $2 \%$ not defined. Table A.1 shows that a large number of the observations are spectral scans that cover most and at least half of the full 5-17 $\mu \mathrm{m}$ spectral range of the $\mathrm{CVF}$.

\section{A.2. Data products}

Results of the processing have been written into FITS files which can be retrieved from the ISO archive (http://www.iso.vilspa.esa.es) as Highly Processed Data Products (HPDP) under the name Mid-IR Spectro Imaging ISOCAM CVF Observations. In this section, we give a description of the FITS files.

The general header of the file contains basic information about the observation: object and pointing coordinates, date and time of the observation. For the 6" pixel observations, the header might include the keywords "RA corr" and "Dec corr" which are the proposed (RA, Dec) coordinates corrected for the mean 2 pixel shift when the vignetting pattern is shifted to the left (right edge column under illuminated). For the $3^{\prime \prime}$ observations, the coordinate correction needs to be computed by the user from the camera angle provided in the header after assessment of the vignetting pattern (see Sect. 4). The first extension header has information about the data columns and their physical units. These data columns provide:

Basic information for each plane: the pixel field of view (pfov) and the wavelength in microns (wave).

Various vectors used in the data reduction useful for scientific analysis, in particular to quantify systematic errors:

- Flag serves to separate wavelength scanning directions and the two CVF segments from filter observations (flag is 0 and 1 for CVF1 scans up and down in wavelength, 2 and 3 for CVF2 scans up and down);

- Zodi Vector, the zodiacal emission from the DIRBE model ( $Z_{\text {mod }}$ in Sect. 3). This vector is useful for estimating the
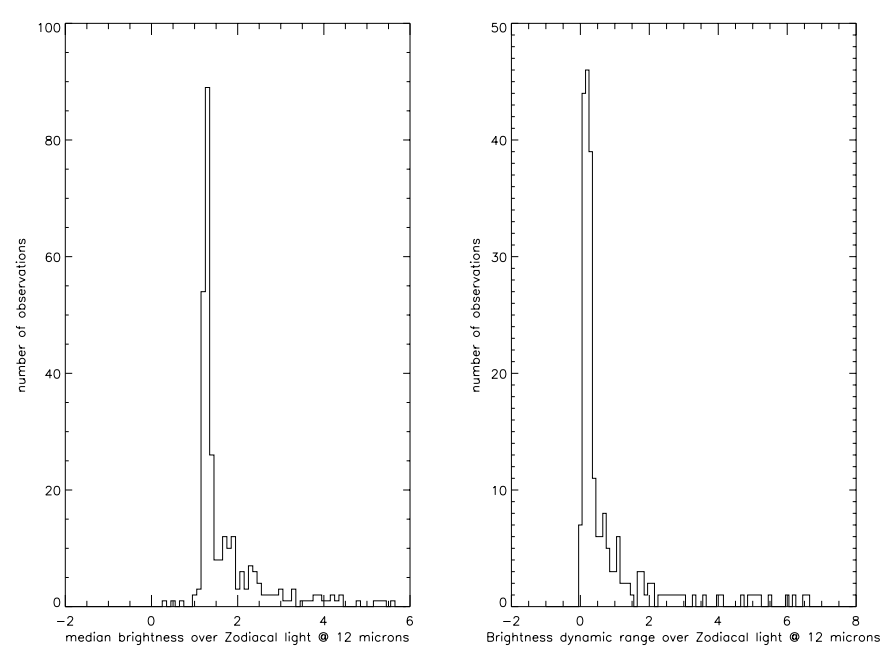

Fig. A.1. Histogram of the median brightness (left) and the dynamic range of the extended emission (right) computed over the observations field of view at $12 \mu \mathrm{m}$ and normalized by the zodiacal emission. The dynamic range is the difference between maximum and minimum brightness after smoothing the data by a $5 \times 5$ pixels window. For a large majority of the observations, the mean brightness and dynamic range are smaller or comparable to the zodiacal emission. The dynamic range has been computed only for the $6^{\prime \prime}$ and $12^{\prime \prime}$ pixel observations.

Table A.1. Statistics of CVF Observations.

\begin{tabular}{lccc}
\hline \hline pixel (") & Step & & Back and forth \\
\hline 1.5 & 1 & 13 & 2 \\
1.5 & 2 & 18 & 2 \\
3 & 1 & 24 & 0 \\
3 & 2 & 21 & 0 \\
6 & 1 & 148 & 18 \\
6 & 2 & 63 & 19 \\
12 & 1 & 16 & 1 \\
12 & 2 & 3 & 3 \\
\hline
\end{tabular}

For each pixel size, the third column gives the number of observations that cover more than half of the 5-17 $\mu \mathrm{m} \mathrm{CVF}$ wavelength range with full spectral sampling (step =1), and sampling every other CVF step $($ step $=2)$. The last column gives the number of these observations performed by scanning both up and down in wavelengths.

systematic uncertainty in continuum emission associated with the zodiacal light subtraction;

- Stray Vec, the vector used in the stray light correction $(B(\lambda) *(1+S(\lambda))$ in Sect. 2.2). In the case of 1.5 and $3^{\prime \prime}$ pixels this vector is not relevant since there is no stray light correction; Stray Vec then only the repeats the zodiacal light (Zodi vector above).

A set of cubes that each give the result of the data reduction at various intermediary stages of the data reduction prior to the projection on celestial coordinates:

- Cube Cal: the calibrated data cube corrected for dark, glitches, and transients;

- Cube Sigma: noise cube for Cube Cal computed by comparing consecutive frames at one given wavelength. Cube 
Sigma provides a good estimate of the spectral noise. From this estimate, the spatial noise may thus be computed from the formula given in Sect. 7.2.1;

- Cube Cal Flat: the flat fielded calibrated data cube that is the data product matching the final standard pipeline result in the ISO archive;

- Cube Cal Zodi: the calibrated data cube after stray light correction and zodiacal light subtraction. This cube is not flat fielded;

- Cube: Cube Cal Zodi flat fielded, plus the wavelength correction applied for 6 " pixel observations with full spectral sampling. This is the final cube before projection onto sky coordinates. For the $6^{\prime \prime}$ and $12^{\prime \prime}$ pixels, the ISO point spread function is ill sampled and the interpolation onto celestial coordinates smears the signal somewhat. In some cases it is thus better to use this cube than the final reprojected cube.

The FITS image has a second extension, which contains the final CVF data cube reprojected onto equatorial coordinates, taking into account the optical distortion of the camera with its header. For most scientific applications this is the only data cube necessary. Brightness is in all cubes expressed in MJy/sr. 